\title{
Comparison between X-Ray Radiography Image Fusion Algorithms Used in Medical Applications
}

\author{
Mohamed S. El_Tokhy \\ Electrical Engineering Department, College of Engineering \\ Aljouf University \\ Aljouf, KSA \\ engtokhy@gmail.com \\ Ibraheem M. Fayed \\ Electrical Engineering Department, College of Engineering \\ Aljouf University \\ Aljouf, KSA \\ imfayed@ju.edu.sa
}

\begin{abstract}
Medical diagnoses methods are very important to determine the reasons of diseases. In this paper different algorithms are introduced to make image fusion using $x$-ray radiography chest image. This fusion is done for front view image and side view image. These algorithms are multiplication factors (MF), multi-resolution singular value decomposition (MSVD), dual tree complex wavelet transform, and discrete stationary wavelet transform (SWT). These algorithms are considered using six different methods. These methods being averaging, max coefficient, block bias based on largest magnitude, energy, block bias based on largest contrast and bias methods. A comparison is accomplished using peak signal to noise ratio (PSNR), mean square error (MSE), entropy, and measure of structural similarity (SSIM). The obtained results confirms that applying the fusion algorithms introduces better results for the four algorithms and enhances the performance characteristics of these algorithms more than using front view image only or side view image only. Furthermore, we concluded that the conventional MF algorithm is superior other three algorithms for most of statistical characteristics.
\end{abstract}

Keywords- image processing, fusion algorithm, and radiography.

\section{INTRODUCTION}

+ Biomedical image processing is a rapidly growing area of research from last two decades. Availability of numerous kinds of biomedical sensors has increased the interest of researchers and scientists in this field. X-ray, ultrasound, magnetic resonance imaging (MRI) and computed tomography (CT) are a few examples of biomedical sensors. These sensors are used for extracting clinical information, which are generally complementary in nature. For example, $\mathrm{X}$-ray is widely used in detecting fractures and abnormalities in bone position, CT is used in tumor and anatomical detection and MRI is used to obtain information among tissues. Similarly, other functional imaging techniques like functional magnetic resonance imaging (MRI), positron emission tomography (PET), and single positron emission computed tomography (SPECT) provide functional and metabolic information. Hence, none of these modalities is able to carry all relevant information in a single image. Therefore, multimodal fusion is required to obtain all possible relevant information in a single composite image [1]. The fusion of data for medical imaging has become a central issue in such biomedical applications as imageguided surgery and radiotherapy [2]. Image fusion is a process to combine information from multiple images of the same scene [3], [4]. The result of image fusion will be a new image which is more suitable for human and machine perception or further tasks of image processing such as image segmentation, feature extraction and object recognition [3]. There are two basic requirements for image fusion [1].

- Fused image should possess all possible relevant information contained in the source images;

- Fusion process should not introduce any artifact, noise or unexpected feature in the fused image.

Image Fusion is one of the important and preprocessing steps in digital image reconstruction [5]. The objective of image fusion is to better the quality of fused images, extract all the useful information from the source images and do not introduce artifacts or inconsistencies which will distract human observers. Many algorithms have been developed for fusion of medical images as reported in the literature [5]. Despite the significant research conducted on this topic, the development of efficient medical image fusion method is still a big challenge for the researchers [5]. This comparison is accomplished using peak signal to noise ratio (PSNR), mean square error (MSE), entropy, and measure of structural similarity (SSIM). 


\section{IMAGE FUSION ALGORITHMS}

The fusion process should preserve all relevant information in the fused image, should reduce noise and should suppress any artifacts in the fused image [6], [7].

Image fusion is the process of integrating all relevant and complementary information from different source images into a single composite image without introducing any artifact or noise [1]. Image fusion can be performed at three levels; pixel level, feature level and decision level [1], [3]. Pixel level fusion deals with information associated with each pixel and fused image can be obtained from the corresponding pixel values of source images [1]. In feature level fusion, source images are segmented into regions and features like pixel intensities, edges or texture, are used for fusion. Decision level fusion is a high level fusion which is based on statistics, voting, fuzzy logic, prediction and heuristics [1].

In the field of image fusion, pixel-level fusion becomes the primary method since it can preserve original information of source images as much as possible, and the algorithms are computationally efficient and easy to implement, the most image fusion applications employ pixel level based method [3]. There are three commonly used methods of pixel-level image fusion, including simple image fusion [3] (such as linear weighted average, HPF (high-pass-filter), HIS (intensity hue-saturation), PCA (principal component analysis)), pyramid-based decomposition image fusion (such as Laplace pyramid decomposition, ratio pyramid) and wavelet transform image fusion [3]. Recently, wavelet transform becomes an important aspect of image fusion research with the merits of multi-scale and multi-resolution [3].

Therefore, the radiography chest image fusion from different views is taken as an example to introduce better diagnoses. Therefore, three algorithms are evaluated and used for this purpose.

\section{A. X-ray radiography chest image fusion based on multiplication factor}

The fusion of radiography chest images can be realized in successive steps as depicted in Fig. 1. This algorithm is based on a MATLAB routine implementing the image fusion algorithm in [8]. In this algorithm, we combine two X-ray radiography chest images. One of the advantages of this algorithm, It supports both gray and color images. The basic idea of this algorithm depends on factor that is lies between zero and one. This factor can be varied to vary the proportion of mixing of each image. Therefore, there are three different cases dependent on this factor $(\mathrm{F})$ :

$$
F= \begin{cases}=0.5 & \text { Equal Mixing } \\ \prec 0.5 & \text { Side View Image Contribution } \\ \succ 0.5 & \text { Front View Image Contribution }\end{cases}
$$

The side view image is multiplied by this factor. However, the front end image was multiplied by (1-F). Both images are added to obtain the fused image. The contribution of both images based on the value of this factor. If the value of the factor is equal to 0.5 , contribution of side view and front view images are identical. Contribution of side view image is larger as this value smaller than 0.5. However, contribution of front view image is larger as this value larger than 0.5 .

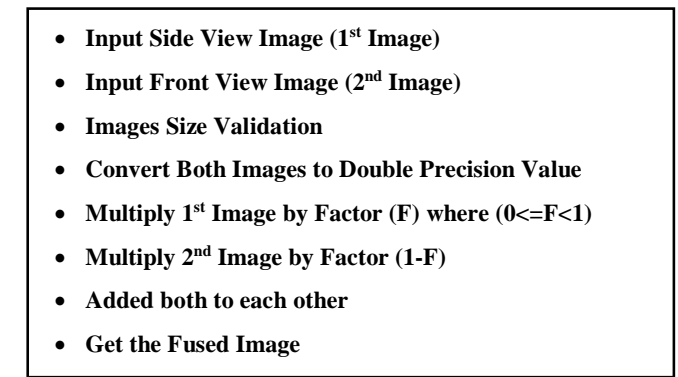

Figure 1. X-ray radiography chest image fusion algorithm using multiplication factor

\section{B. X-ray radiography chest image fusion based on multi-resolution singular valuedecomposition}

Multi-resolution singular value decomposition (MSVD) is very similar to wavelets transform, where signal is filtered separately by low pass and high pass finite impulse response (FIR) filters and the output of each filter is decimated by a factor of two to achieve first level of decomposition [9]. The decimated low pass filtered output is filtered separately by low pass and high pass filter followed by decimation by a factor of two provides second level of decomposition. The successive levels of decomposition can be achieved by repeating this procedure. The idea behind the MSVD is to replace the FIR filters with singular value decomposition (SVD) [9]. An algorithm for image fusion based on MSVD is studied as depicted in Fig. 2. This algorithm is based on a MATLAB routine implementing the image fusion algorithm in [9].

The images to be fused I1 and I2 are decomposed into $\mathrm{L}(\mathrm{l}=1,2, \ldots, \mathrm{L})$ levels using MSVD. At each

decomposition level $(1=1,2, \ldots, \mathrm{L})$, the fusion rule will select the larger absolute value of the two MSVD detailed coefficients, since the detailed coefficients correspond to sharper brightness changes in the images [9]. These coefficients are fluctuating around zero. At the coarest level $(1=\mathrm{L})$, the fusion rule take average of the MSVD approximation coefficients since the approximation coefficients at coarser level are the smoothed and subsampled version of the original image. Similarly, at each decomposition level $(1=1,2, \ldots, \mathrm{L})$, the fusion rule take the average of the two MSVD eigen matrices [9]. 


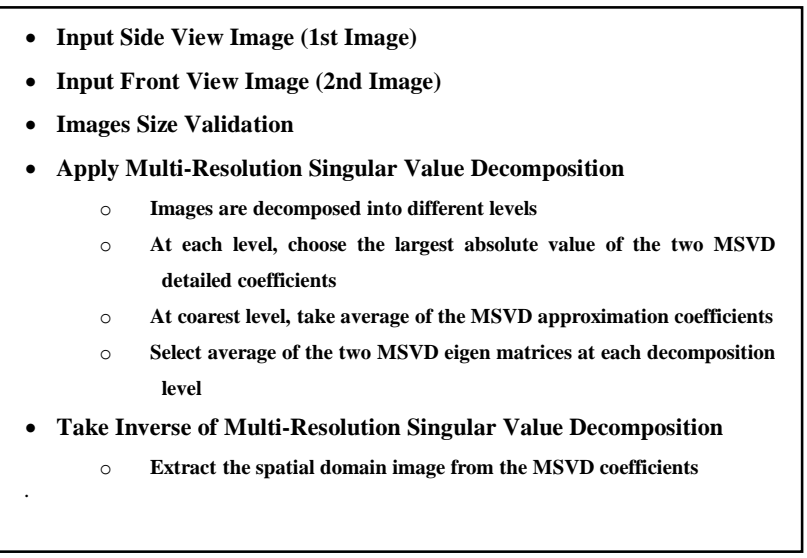

Figure 2. X-ray radiography chest image fusion algorithm using multiresolution singular value decomposition

C. X-ray radiography chest image fusion based on dual tree complex wavelet transform

Another algorithm for image fusion based on dual-tree complex wavelet transform is studied as depicted in Fig. 3. This algorithm is based on a MATLAB routine implementing the image fusion algorithm in [10]. There are two representations of the 2D dual-tree wavelet transform; the real 2D dual-tree DWT and complex 2D dual-tree DWT. The dual-tree complex wavelet transform (DTCWT) is a relatively recent enhancement to the DWT, with important additional properties: nearly shift-invariant and directionally selective (useful in two and higher dimensions) [11], [12]. In the dual-tree implementation of decomposition and reconstruction, two parallel DWTs with different low-pass and high-pass filters in each scale are used as can be seen in Fig. 4 [11]. The two DWTs use two different sets of filters, with each satisfying the perfect reconstruction condition.

One of the advantages of the dual-tree complex wavelet transform is that it can be used to implement 2D wavelet transforms that are more selective with respect to orientation than is the separable 2D discrete wavelet transform (DWT) [10], [11]. The complex 2D dual-tree DWT gives rise to wavelets in six distinct directions. In each direction, one of the two wavelets can be interpreted as the real part of a complex-valued 2D wavelet, while the other wavelet can be interpreted as the imaginary part of a complex-valued 2D wavelet. The complex 2D dual-tree is implemented as four critically-sampled separable 2D DWTs operating in parallel [10]. However, different filter sets are used along the rows and columns [10], [11]. Furthermore, the sum and difference of sub-band images is performed to obtain the oriented wavelets [10].

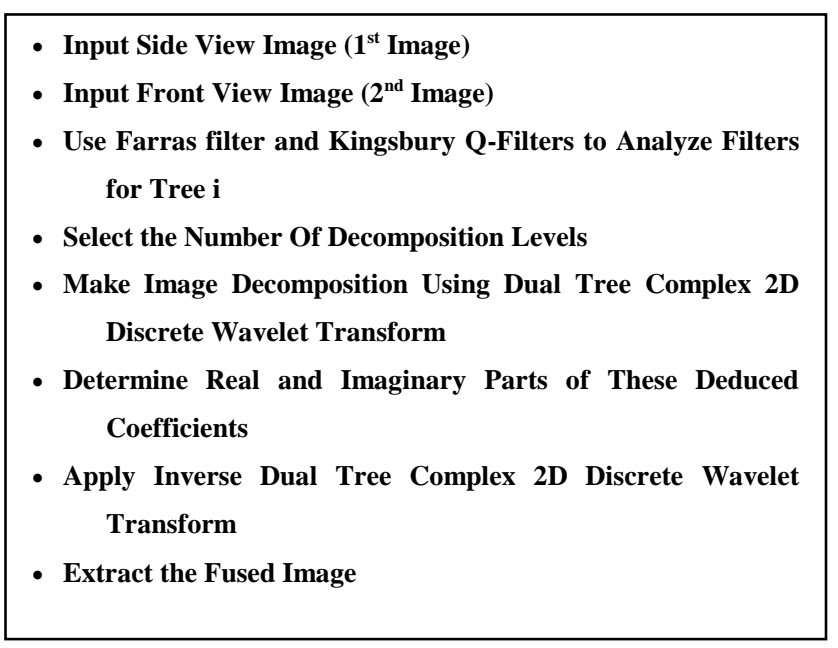

Figure 3. X-ray radiography chest image fusion algorithm using dual tree complex discrete wavelet transforms

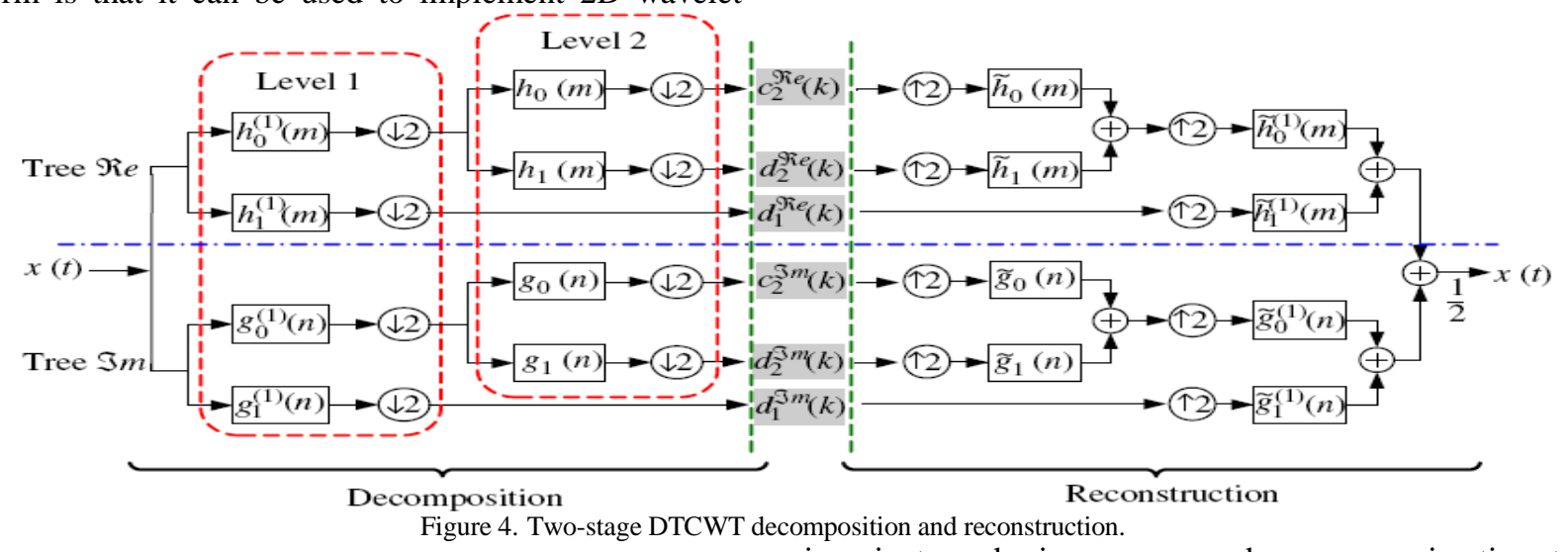

\section{X-ray radiography chest image fusion based on discrete stationary wavelet transform}

Recently, the DWT has become a powerful tool for multiscale image fusion [13]. Stationary wavelet transform (SWT) is similar to discrete wavelet transform (DWT) but the only process of down-sampling is suppressed that means the SWT is translation-invariant [4]. It is redundant, shift invariant, and gives a more dense approximation to the continuous wavelet transform than discrete wavelet transforms [14]. The discrete wavelet transform (DWT) is a common tool for image fusion, but the result could contain the artifacts near the edges. This impairment is addressed by the models based on stationary wavelet transform (SWT), curvelet transform and non-sampled contourlet (NSCT). Recent studies show that both SWT and NSCT turn out to be 
the more suitable fusion approaches because of their shiftinvariant [15]. The way to restore the translation invariance is to average some slightly different DWT, called decimated DWT, to define the stationary wavelet transform (SWT). Let us recall that the DWT basic computational step is a convolution followed by decimation. The decimation retains even indexed elements. But the decimation could be carried out by choosing odd indexed elements instead of even indexed elements [6].

The SWT algorithm is very simple and is close to the DWT one [4], [6], [13]. More precisely, for level 1, all the decimated DWT for a given signal can be obtained by convolving the signal with the appropriate filters as in the DWT case but without down sampling. Then, the approximation and detail coefficients at level 1 are both of size $\mathrm{N}$, which is the signal length. The general step $\mathrm{j}$ convolves the approximation coefficients at level $\mathrm{j}-1$, with up sampled versions of the appropriate original filters, to produce the approximation and detail coefficients at level $\mathrm{j}$ [6].

Figure 5 and Figure 6 Show the fusion process for the front view image and the side view image to get better image with more details.

\section{- Load Side View Image ( $1^{\text {st }}$ Image $)$}

- Load Front View Image ( $2^{\text {nd }}$ Image)

- Perform Image decomposition using discrete stationary wavelet transforms into different

levels

- Display the approximation and detail coefficients at the different levels

- Reconstruct the fused image from these coefficients by Applying the Inverse of discrete

\section{- stationary wavelet transforms}

Figure 5. X-ray radiography chest image fusion algorithm using discrete stationary wavelet transforms

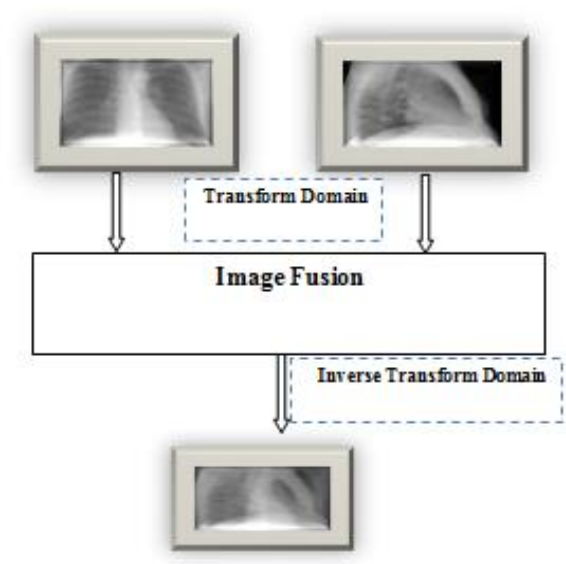

Figure 6. Image fusion algorithm based on DST and FFT

\section{RESULTS AND DiscussioA}

Comparison between these algorithms is of major concern. Therefore, this comparison study is done between the considered algorithms using different statistical evaluation processes. These, statistics are root mean square error (RMSE), mean absolute error (MAE), signal to noise ratio (SNR), and peak signal to noise ratio (PSNR). The statistical measurements between fused image and both front image and side image for multiplication, MSVD, DTCWT and SWT algorithms are depicted in Tables 1-4. We noticed that MSE between fused image and side view image is lower than that between fused image and front one for most algorithms. Also, SNR and PSNR between fused image and side view image are larger than that between fused image and front one for most algorithms.

TABLE I STATISTICAL SUREMENTS FOR MULTIPLICATION FACTOR OF FRONT AND SIDE VIEW IMAGE

\begin{tabular}{|c|c|c|l|c|c|c|}
\hline & MSE & PSNR & Entropy & SNR & RMSE & MAE \\
\hline $\begin{array}{c}\text { MF } \\
\text { for } \\
\text { Front } \\
\text { Image }\end{array}$ & 39.7887 & 32.1332 & 7.477 & 0.00670 & 6.3078 & 0.1952572 \\
\hline $\begin{array}{c}\text { MF } \\
\text { Side } \\
\text { Image }\end{array}$ & 22.8838 & 34.5355 & 7.477 & 1.02669 & 4.7837 & 0.1952572 \\
\hline
\end{tabular}

TABLE II STATISTICAL MEASUREMENTS FOR MSVD OF FRONT AND SIDE VIEW IMAGE

\begin{tabular}{|c|c|c|c|c|c|c|}
\hline $\begin{array}{c}\text { MSVD } \\
\text { for } \\
\text { Front } \\
\text { Image }\end{array}$ & 0.029093 & 63.4929 & 6.8489 & 0.15495 & 0.17057 & 0.13495 \\
\hline $\begin{array}{c}\text { MSVD } \\
\text { Side } \\
\text { Image }\end{array}$ & 0.028496 & 63.5829 & 6.8489 & 0.69227 & 0.16881 & 0.13426 \\
\hline
\end{tabular}

TABLE III STATISTICAL MEASUREMENTS FOR DTCWT OF FRONT AND SIDE VIEW IMAGE

\begin{tabular}{|c|c|c|c|c|c|c|}
\hline & MSE & PSNR & Entropy & SNR & RMSE & $\begin{array}{c}\text { MA } \\
\text { E }\end{array}$ \\
\hline $\begin{array}{c}\text { DTCWT } \\
\text { for } \\
\text { Front } \\
\text { Image }\end{array}$ & 0.082808 & 58.9501 & 6.6614 & 2.54017 & 0.28776 & $\begin{array}{c}0.208 \\
25\end{array}$ \\
\hline $\begin{array}{c}\text { DTCWT } \\
\text { for Side } \\
\text { Image }\end{array}$ & 0.17579 & 55.6809 & 6.6614 & 3.07749 & 0.41927 & $\begin{array}{c}0.319 \\
62\end{array}$ \\
\hline
\end{tabular}


ICIT 2015 The $7^{\text {th }}$ International Conference on Information Technology

doi:10.15849/icit.2015.0025 C ICIT 2015 (http://icit.zuj.edu.jo/ICIT15)

TABLE IV STATISTICAL MEASUREMENTS FOR SWT OF FRONT AND SIDE VIEW IMAGE

\begin{tabular}{|c|c|c|c|c|c|c|}
\hline & MSE & PSNR & Entropy & SNR & RMSE & MAE \\
\hline 总苜泀 & 0.029068 & 63.4966 & 6.8945 & 0.68034 & 0.17049 & 0.13479 \\
\hline 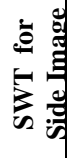 & 0.028382 & 63.6003 & 6.8945 & 0.14631 & 0.16847 & 0.13388 \\
\hline
\end{tabular}

Comparison between multiplication factor (MF), MSVD, DTCWT, and SWT(L1) algorithms based on the mentioned statistics before and after applying the fast lifting transform are depicted in Tables 1-2, respectively. From these tables, the discrete stationary wavelet transform (SWT) and MF achieve the best performance for most characteristics before and after applying the fast lifting transform.

TABLE V X-RAY RADIOGRAPHY CHEST IMAGE FUSION QUALITY BASED ON THE MF, MSVD, DTCWT AND SWT(L1) ALGORITHMS BEFORE APPLYING THE FAST LIFTING TRANSFORM

\begin{tabular}{|c|c|c|c|c|}
\hline Statistics & MF & MSVD & DTCWT & SWT(L1) \\
\hline RMSE & 0.2473732 & 0.2216372 & 0.2858792 & 0.1704952 \\
\hline FE (\%) & 46.9770992 & 53.3529622 & 54.2895132 & 30.0690352 \\
\hline MAE & 0.1952572 & 0.1852432 & 0.2225302 & 0.1347882 \\
\hline CORR & 0.8892772 & 0.8900532 & 0.8124702 & 0.9512592 \\
\hline SNR & 6.5622762 & 5.4568292 & 5.3056812 & 10.4376102 \\
\hline PSNR & 54.2312812 & 54.7083792 & 53.6029832 & 55.8476892 \\
\hline MI & 1.0807172 & 1.0016432 & 1.0977672 & 1.0245702 \\
\hline QI & 0.1177012 & 0.1208092 & 0.2189232 & 0.5043482 \\
\hline SSIM & 0.9927212 & 0.9935042 & 0.9895282 & 0.9968282 \\
\hline
\end{tabular}

TABLE VI X-RAY RADIOGRAPHY CHEST IMAGE FUSION QUALITY BASED ON THE MF, MSVD, DTCWT, AND SWT(L1) ALGORITHMS AFTER LIFTING TRANSFORM

\begin{tabular}{|c|c|c|c|c|}
\hline Statistics & MF & MSVD & DTCWT & SWT(L1) \\
\hline RMSE & 0.2473732 & 0.2471542 & 0.2858992 & 0.1684962 \\
\hline FE (\%) & 46.9770992 & 46.9200632 & 54.2754102 & 31.5915932 \\
\hline MAE & 0.1952572 & 0.1952642 & 0.2227982 & 0.1339862 \\
\hline CORR & 0.8892772 & 0.8893332 & 0.8125132 & 0.9492692 \\
\hline SNR & 6.5622762 & 6.5728282 & 5.3079382 & 10.0085692 \\
\hline PSNR & 54.2312812 & 54.2351182 & 53.6026732 & 55.8989042 \\
\hline MI & 1.0807172 & 1.0820882 & 1.0951482 & 1.1495912 \\
\hline QI & 0.1177012 & 0.1214162 & 0.2188072 & 0.4466332 \\
\hline SSIM & 0.9927212 & 0.9927562 & 0.9895242 & 0.9967232 \\
\hline
\end{tabular}

\section{CONCLUSION}

Different algorithms are evaluated to make image fusion using $\mathrm{x}$-ray radiography chest image. This fusion is done for front view image and side view image. These algorithms are named multiplication factor (MF), multi-resolution singular value decomposition (MSVD), dual tree complex wavelet transform, and discrete stationary wavelet transform (SWT). These algorithms are evaluated using statistical measurements. The obtained results confirm that MSE between fused image and side view image is lower than that between fused image and front one for most algorithms. Also, SNR and PSNR between fused image and side view image are larger than that between fused image and front one for most algorithms. Therefore, using the fused image and side view image will have more details than front image with fused image. Furthermore, using fused image and side view difference processing with SWT and MF makes diagnosis of respiratory diseases more accurate.

\section{REFERENCES}

[1] Rajiv Singh, Ashish Khare, "Fusion of multimodal medical images using Daubechies complex wavelet transform-A multiresolution approach", Information Fusion, Vol. 19, pp. 49-60, 2012 [http://dx.doi.org/10.1016/j.inffus.2012.09.005].

[2] Zhiping Xu, "Medical image fusion using multi-level local extrema", Information Fusion, Vol. 19, pp.38-48, 2013 [http://dx.doi.org/10.1016/j.inffus.2013.01.001]

[3] S. V. More, and S. D. Apte, "Pixel-Level Image Fusion Using Wavelet Transform", International Journal of Engineering Research \& Technology (IJERT), Vol. 1, Vol. 5, 2012.

[4] Pusit Borwonwatanadelok, Wirat Rattanapitak and Somkait Udomhunsakul, "Multi-Focus Image Fusion based on Stationary Wavelet Transform and extended Spatial Frequency Measurement", International Conference on Electronic Computer Technology, 2009.

[5] Navneet Kaur, Jaskiran Kaur, "A Novel Method For Pixel Level Image fusion Based on Curvelet Transform", International Journal of Research in Engineering and Technology (IJRET), Vol. 1, No. 1, 2013.

[6] Kanagaraj Kannan, Subramonian Arumuga Perumal, Kandasamy Arulmozhi, "Optimal decomposition level of discrete, stationary and dual tree complex wavelet transform for pixel based fusion of multifocused images", Serbian Journal of Electrical Engineering, Vol. 7, No. 1, May 2010, 81-93

[7] Y. Chai, H.F. Li, M.Y. Guo, "Multifocus image fusion scheme based on features of multiscale products and PCNN in lifting stationary wavelet domain", Optics Communications, Vol. 284, pp. 1146-1158, 2011.

[8] Athi, Matlab Central, "mixing or combining two images (image fusion)", Mathwork, 2009.

[9] V.P.S. Naidu, "Image Fusion Technique using Multi-resolution Singular Value Decomposition", Defence Science Journal, Vol. 61, No. 5, , pp. 479-484, 2011.

[10] V.P.S. Naidu, "2D dual tree complex wavelet transform", http://taco.poly.edu/WaveletSoftware/, 2013.

[11] Yanxue Wang, Zhengjia He, Yanyang Zi, "Enhancement of signal denoising and multiple fault signatures detecting in rotating machinery using dual-tree complex wavelet transform", Mechanical Systems and Signal Processing, Vol. 24, pp. 119-137, 2010.

[12] Ivan W. Selesnick, Richard G. Baraniuk, and Nick G. Kingsbury, "The Dual-Tree Complex Wavelet Transform", IEEE Signal Processing Magazine, pp. 123-151, 2005.

[13] Hailiang Shi, Min Fang, "Multi-focus Color Image Fusion Based on SWT and IHS", Fourth International Conference on Fuzzy Systems and Knowledge Discovery, 2007.

[14] Cungui Cheng, Jia Liu, Wenqing Cao, Renwei Zheng, Hong Wang, Changjiang Zhang, "Classification of two species of Bidens based on discrete stationary wavelet transform extraction of FTIR spectra 
ICIT 2015 The $7^{\text {th }}$ International Conference on Information Technology doi:10.15849/icit.2015.0025 C ICIT 2015 (http://icit.zuj.edu.jo/ICIT15)

combined with probability neural network", Vibrational Spectroscopy, Vol. 54, No. 1, pp. 50-55, 2010.

[15] Zhou Zeming, Jiang Lin, Wang Jin, Zhang Peng, Yang Pinglv, "Image Fusion by Combining SWT and Variational Model", 4th International Congress on Image and Signal Processing, Vol. 4, 2011. 